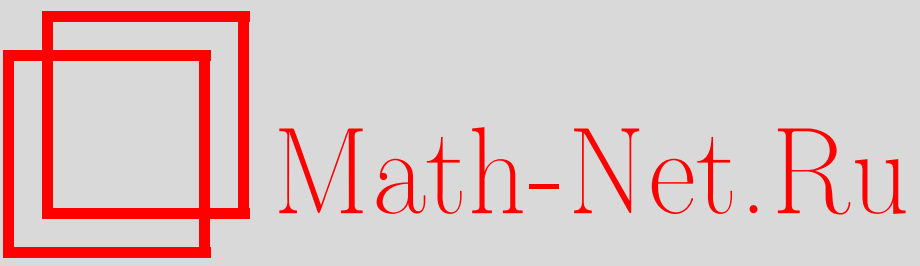

Л. А. Зайцева, Термодинамическая энергия полуограниченных твердых тел с двумерным газом горячих электронов вблизи поверхности, ТМФ, 2001, том 127, номер 2, 336-352

DOI: https://doi.org/10.4213/tmf461

Использование Общероссийского математического портала Math-Net.Ru подразумевает, что вы прочитали и согласны с пользовательским соглашением

http: //www . mathnet.ru/rus/agreement

Параметры загрузки:

IP : 54.147 .182 .235

26 апреля 2023 г., 18:21:38 


\section{ТЕРМОДИНАМИЧЕСКАЯ ЭНЕРГИЯ ПОЛУОГРАНИЧЕННЫХ ТВЕРДЫХ ТЕЛ С ДВУМЕРНЫМ ГАЗОМ ГОРЯЧИХ ЭЛЕКТРОНОВ ВБЛИЗИ ПОВЕРХНОСТИ}

Получены выражения для термодинамической энергии полуограниченных твердых тел различной ширины с тонким слоем горячих вырожденных электронов вблизи свободной поверхности. Показано, что в тонких пленках этой энергией является энергия возбуждения горячих электронов, в толстых пленках - неравновесная энергия рэлеевских волн, в больших образцах - равновесная энергия объемных фононов. Все расчеты проводились с помощью диаграммной техники Келдыша.

\section{1. ВВЕДЕНИЕ}

Предметом исследования в данной работе являются полуограниченные кристаллы с тонким слоем горячих вырожденных электронов вблизи свободной поверхности. Такие системы реализуются в полупроводниковых приборах. Это могут быть, например, полупроводники с гетеропереходами (при контакте двух различных по химическому составу образцов) или с инверсионными слоями (с повышенной концентрацией неосновных носителей тока вблизи гранищы с металлом).

Термодинамической энергией кристалла является часть его полной средней энергии, зависящая от температуры [1]. В рассматриваемых образцах она определяется энергией фононов и энергией возбуждения электронов с учетом электрон-фононного взаимодействия. Не зависящая от температуры часть полной средней энергии представляет собой энергию нулевых колебаний решетки и энергию вырождения электронов ${ }^{1}{ }^{1}$.

Электроны принято называть горячими, если их средняя энергия больше средней энергии электронов в состоянии равновесия с решеткой. У электронов, нагретых лазером или током, в результате электрон-электронного взаимодействия может установить-

\footnotetext{
1) При температуре среды $T$ ниже температуры Дебая $\Theta$ эти энергии соответственно больше энергии фононов и энергии возбужения электронов. Однако такие важнейшие макрохарактеристики веществ, как, например, теплоемкость, определяются их термодинамической энергией.
}

${ }^{*}$ Институт химической физики им. Н. Н. Семенова РАН, Москва, Россия. E-mail: zaitseva@center.chph.ras.ru 


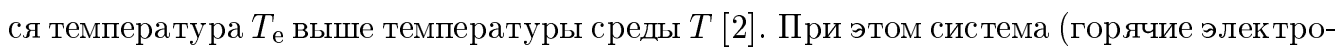
ны плюс фононы) в целом остается определенное время неравновесной.

Тонкий слой электронов можно считать двумерным, если его толщина $d \lesssim l$, где $l$ - характерная длина волны фононов. Расстоянием слоя электронов от поверхности $z_{0}$ можно пренебречь, если $z_{0} \lesssim l$. Как известно, при $T_{\mathrm{e}}<\mu$, где $\mu$ - энергия Ферми, электроны вырожденны и представляют собой почти идеальный газ.

Сушественное различие в акустических свойствах контактируюших вешеств или плохой механический контакт затрудняют прохождение акустических фононов через гранищу раздела. Компоненты тензора напряжений на такой поверности по нормали к ней можно считать равными нулю. Определенную так поверхность принято называть свободной [3]. В данной работе будут рассматриваться полуограниченные кристаллы различного размера. При этом предполагается, что поверхность, противоположная свободной, у больших образцов удалена на бесконечность. Это означает, что их размер $L_{z} \gg l$. В пленках с толшинами $L_{z}^{0} \lesssim l$ вторая поверхность плотно контактирует с акустически согласованной средой. Размеры $L_{x}, L_{y} \gg l$ в образцах с любым $L_{z}$.

Надежных расчетов неравновесных макрохарактеристик ограниченных кристаллов не так много. Наличие поверхности у неравновесной системы значительно усложняет такие расчеты. Это связано с присутствием вблизи свободной поверхности рэлеевских волн [3], [4]. Кроме того, сушествуюшие наряду с ними объемные волны частично меняют свою поляризацию при отражении от такой поверхности. Термодинамические характеристики равновесных полуограниченных кристаллов различной толшины (например, теплоемкость, средняя энергия) вычислялись ранее [5]-[7]. Получены формулы для термодинамической энергии неограниченных образцов с равнораспределенными в объеме горячими электронами [8]. Более многочисленны расчеты кинетических характеристик горячих электронов, которые определяются интегралом столкновений в кинетическом уравнении для электронной функции распределения. Так, в работе [9] вычислены моцности потерь (темп релаксации энергии и температуры) горячих электронов, равнораспределенных в объеме неограниченного образца вследствие их рассеяния на деформационном потенциале решетки. В работе [10] найдены условия, при которых темп релаксации энергии двумерного газа горячих электронов из-за такого же рассеяния значительно снижен вблизи свободной поверхности полуограниченного кристалла.

В данной работе вычисляется полная средняя колебательная энергия полуограниченных кристаллов различной толщины с двумерным газом горячих электронов вблизи свободной поверхности. Вычисления проводятся, как и в работах [7], [8], методом диаграммной техники Келдыша [11], [12]. В рассматриваемом случае важно, что функции Келдыша квадратичны по смешениям. Это избавляет от необходимости следить за эволюцией векторов смешений при отражении от границ, как это обычно делается [5], [6]. Вблизи поверхности векторы поляризации объемных волн теряют строгую ориентацию относительно направлений своих волновых векторов. В этой ситуации вектор смешений среды может быть представлен в виде линейной комбинации продольных (l) и поперечных (t) смещений. Их удельный вклад в общий вектор смешений определяется граничными условиями к уравнениям движения упругой среды [3]. Техника Келды- 
ша [11], [12] применима к неограниченным средам. В данной работе получены функции Келдыша свободных фононов в полуограниченных кристаллах. С этой целью используются функции Грина уравнений движения упругой среды с учетом обрыва упругих констант на поверхности $(z=0)$ и затухания решений при $z \rightarrow \infty$. Эти функции были получены в работе [13], где с их помощью вычислялось затухание рэлеевских волн на шероховатостях свободной поверхности. Функции Келдыша свободных электронов в слое будут представлены в виде произведения двумерных функций Келдыша и модельных функций от переменных $z$, введенных в работе [10]. Потенциал взаимодействия фононов с электронами будем считать деформационным.

Сушественно, что техника Келдьша применима для расчета макрохарактеристик неравновесных систем при любых температурах подсистем, включая нулевые. Это позволяет выделить из полной средней энергии кристалла с горячими электронами термодинамическую энергию. Как показано в [8], термодинамической энергией неограниченного образца с распределенными в объеме горячими электронами при $T<T_{\mathrm{e}} \ll \Theta$ является неравновесная энергия фононов. Вследствие нагрева части фононов до температуры $T_{\mathrm{e}}$ из-за деформационного взаимодействия с горячими электронами эта энергия зависит от $T_{\mathrm{e}}$ и $T$. При $T_{\mathrm{e}} \gg T$ неравновесная энергия фононов оказывается больше энергии свободных фононов, зависяшей от $T$. При малой плотности электронов неравновесная энергия фононов превышает также энергию возбуждения электронов. В данной работе найден относительный вклад неравновесной энергии пространственных и поверхностных волн в полную среднюю энергию полуограниченных кристаллов различной толщины со слоем горячих электронов вблизи свободной поверхности.

\section{2. ПОЛНАЯ СРЕДНЯЯ КОЛЕБАТЕЛЬНАЯ ЭНЕРГИЯ ПОЛУОГРАНИЧЕННОГО КРИСТАЛЛА СО СЛОЕМ ГОРЯЧИХ ЭЛЕКТРОНОВ}

Выразим полную среднюю колебательную энергию решетки изолированного кристалла через удвоенную кинетическую энергию [14] по формуле [8]

$$
E=\left.i \int d \mathbf{r} \frac{\partial^{2}}{\partial t \partial t_{1}} \operatorname{Sp} D_{\alpha \beta}^{-+}\left(X, X_{1}\right)\right|_{X_{1}=X}
$$

где

$$
D_{\alpha \beta}^{-+}\left(X, X_{1}\right)=-i \rho\left\langle\widehat{U}_{\beta}\left(X_{1}\right) \widehat{U}_{\alpha}(X)\right\rangle
$$

- вневременная функция Келдыша [11], [12]. В соотношениях (1), (2) $\rho$-плотность вешества, $X=(\mathbf{r}, t)$, оператор смешений $\widehat{U}_{\alpha}(X)$ точек среды с координатой $\mathbf{r}$ в направлении $\alpha$ учитывает полуограниченность образца и взаимодействие фононов с горячими электронами. Угловые скобки в (2) подразумевают усреднение по числам заполнения электронов и фононов и по их распределениям Гиббса. Ограничимся в $D_{\alpha \beta}^{-+}$первыми неисчезающими членами разложения в ряд по деформационному гамильтониану взаимодействия

$$
\widehat{H}(t)=\lambda \int d \mathbf{r} \psi^{+}(X) \nabla \hat{\mathbf{u}}(X) \psi(X),
$$


где $\lambda$ - константа деформационного взаимодействия в кристаллах кубической симметрии, $\psi^{+}, \psi$ - вторично квантованные операторы электронного поля, $\hat{\mathbf{u}}$ - операторы свободных смешений полуограниченной решетки.

Полуограниченная среда в направлении $z$ неоднородна. Решения уравнений движения такой среды в виде функций Грина получены в [13] в смешанном $\omega, \mathbf{q}, z$-представлении, где $\omega$ - частота, а $\mathbf{q}=\left\{q_{x}, q_{y}\right\}$ - двумерный импульс. Заметим, что в частотном представлении переход от функций Грина к запаздывающим функциям Грина осуществляется простой заменой $\omega$ на $\omega+i \delta$, где $\delta \rightarrow 0$ [14]. Известно [11], [12], что функции Келдыша свободных частиш связаны с запаздываюшими $(\mathrm{R})$ и опережаюшими $(\mathrm{A})$ функциями Грина через распределения Планка и Ферми только в частотно-импульсном представлении. Существенно при этом, что распределение Планка

$$
n(\omega)=\left[\exp \left(\frac{\omega}{T}\right)-1\right]^{-1}
$$

зависит только от частоты $\omega$. Поэтому вьшеупомянутые связи функций Келдьша с функциями Грина справедливы и в смешанном представлении [10].

Выразим энергию (1) через функцию $D_{\alpha \beta}^{-+}\left(\omega, \mathbf{q}, z, z_{1}\right)$. После соответствующих преобразований Фурье получаем, что

$$
\begin{gathered}
E=\frac{L_{x} L_{y}}{(2 \pi)^{3}} \int_{-\infty}^{\infty} d \omega \omega^{2} \int d \mathbf{q} \int_{0}^{L} d z D^{-+}(\omega, \mathbf{q}, z) \\
D^{-+}=\mathcal{D}^{-+}+\Delta \mathcal{D}^{-+}
\end{gathered}
$$

где $D^{-+}(\omega, \mathbf{q}, z)=\left.\operatorname{Sp} D_{\alpha \beta}^{-+}\left(\omega, \mathbf{q}, z, z_{1}\right)\right|_{z_{1}=z}$ и

$$
\mathcal{D}_{\alpha \beta}^{-+}\left(\omega, \mathbf{q}, z, z_{1}\right)=2 n(\omega) \operatorname{Im} \mathcal{D}_{\alpha \beta}^{\mathrm{R}}\left(\omega, \mathbf{q}, z, z_{1}\right)
$$

- вневременная функция Келдыша свободных фононов в полуограниченном пространстве. Энергия свободных акустических колебаний решетки $E_{\mathrm{av}}$, определяемая формулой (5) с функцией (7), вычислена в работе [7]. Выражения для $E_{\text {av }}$ приведены в приложении (формулы (П.13)-(П.16)). Поправка $\Delta E \mathrm{k} E_{\mathrm{av}}$ определяется функцией

$$
\begin{aligned}
\Delta \mathcal{D}_{\alpha \beta}^{-+}(\omega, \mathbf{q}, z)= & \frac{i \lambda^{2}}{(2 \pi)^{3} \rho} \operatorname{Sp} \sum_{\gamma, \delta} \sum_{i, j}(-1)^{\delta_{i j}} \int_{0}^{L} d z_{1}\left|\psi\left(z_{1}\right)\right|^{2} \int_{0}^{L} d z_{2}\left|\psi\left(z_{2}\right)\right|^{2} \times \\
& \times \hat{q}_{\gamma} \hat{q}_{\delta} \mathcal{D}_{\alpha \gamma}^{-i}\left(\omega, \mathbf{q}, z, z_{1}\right) \Pi^{i j}\left(\omega, \mathbf{q}, z_{1}, z_{2}\right) \mathcal{D}_{\delta \beta}^{j+}\left(\omega, \mathbf{q}, z_{2}, z\right) .
\end{aligned}
$$

Здесь при $\gamma, \delta=x, y, z, \nu=x, y, i, j=+,-$ учтено, что

$$
\sum_{\gamma} \mathcal{D}_{\alpha \gamma}^{-i}=\sum_{\nu}-i q_{\nu} \mathcal{D}_{\alpha \nu}^{-i}+\frac{\partial}{\partial z_{1}} \mathcal{D}_{\alpha z}^{-i}, \quad \sum_{\beta} \mathcal{D}_{\beta \delta}^{j+}=\sum_{\nu} i q_{\nu} \mathcal{D}_{\nu \delta}^{j+}+\frac{\partial}{\partial z_{2}} \mathcal{D}_{z \delta}^{j+}
$$

Поляризационные части в (8) имеют вид

$$
\Pi^{i j}(\omega, \mathbf{q})=\int_{-\infty}^{\infty} d \varepsilon \int d \mathbf{p} G^{i j}(\varepsilon, \mathbf{p}) G^{j i}(\varepsilon-\omega, \mathbf{p}-\mathbf{q}),
$$


где $\varepsilon-$ энергия, а $\mathbf{p}=\left\{p_{x}, p_{y}\right\}-$ двумерный импульс электронов. Функции $G^{i j}(\varepsilon, \mathbf{p})$ формально совпадают с выражениями для трехмерных электронных функций Келдыша в работе [12] (формулы (П.1), (П.2) в приложении). Функции $\left|\psi\left(z_{1}\right)\right|^{2}$ в формуле (8) характеризуют распределение электронов в слое в направлении, перпендикулярном поверхности [10].

Поправка $\Delta \mathcal{D}^{-+}(\omega, \mathbf{q}, z)$, как и в работе [8], может быть записана через запаздывающие $\mathcal{D}_{\alpha \beta}^{\mathrm{R}}$ и опережающие $\mathcal{D}_{\alpha \beta}^{\mathrm{A}}$ функции Грина с помощью известных соотношений [11], $[12]$

$$
\begin{gathered}
\sum_{i, j} \mathcal{D}_{\alpha \beta}^{i j}(-1)^{\delta_{i j}}=0, \quad \sum_{i, j} \Pi^{i j}(-1)^{\delta_{i j}}=0, \\
\mathcal{D}_{\alpha \beta}^{\mathrm{R}}=\mathcal{D}_{\alpha \beta}^{--}-\mathcal{D}_{\alpha \beta}^{-+}, \quad \mathcal{D}_{\alpha \beta}^{\mathrm{A}}=\mathcal{D}_{\alpha \beta}^{--}-\mathcal{D}_{\alpha \beta}^{+-} .
\end{gathered}
$$

$\Phi$ ункцию $\Delta \mathcal{D}^{-+}(\omega, \mathbf{q}, z)$ удобно представить в виде суммы $\Delta \widetilde{\mathcal{D}}^{-+}+\Delta \overline{\mathcal{D}}^{-+}$, использовав формулы (7) и (П.5), (П.6) для $\Pi^{i j}$, где

$$
\begin{aligned}
\Delta \widetilde{\mathcal{D}}_{\alpha \beta}^{-+}= & -\frac{2 i \lambda^{2} I_{2}}{(2 \pi)^{3} \rho}\left(n_{\mathrm{e}}-n\right) \sum_{\gamma, \delta} \int_{0}^{L} d z_{1}\left|\psi\left(z_{1}\right)\right|^{2} \int_{0}^{L} d z_{2}\left|\psi\left(z_{2}\right)\right|^{2} \operatorname{Sp} \hat{q}_{\gamma} \hat{q}_{\delta} \mathcal{D}_{\alpha \gamma}^{\mathrm{R}} \mathcal{D}_{\delta \beta}^{\mathrm{A}}, \\
\Delta \overline{\mathcal{D}}_{\alpha \beta}^{-+}= & -\frac{i \lambda^{2}}{(2 \pi)^{3} \rho} n \sum_{\gamma, \delta} \int_{0}^{L} d z_{1}\left|\psi\left(z_{1}\right)\right|^{2} \int_{0}^{L} d z_{2}\left|\psi\left(z_{2}\right)\right|^{2} \times \\
& \times \operatorname{Sp} \hat{q}_{\gamma} \hat{q}_{\delta}\left[i I_{1}\left(\mathcal{D}_{\alpha \gamma}^{\mathrm{R}} \mathcal{D}_{\delta \beta}^{\mathrm{R}}-\mathcal{D}_{\alpha \gamma}^{\mathrm{A}} \mathcal{D}_{\delta \beta}^{\mathrm{A}}\right)+I_{2}\left(\mathcal{D}_{\alpha \gamma}^{\mathrm{R}} \mathcal{D}_{\delta \beta}^{\mathrm{R}}+\mathcal{D}_{\alpha \gamma}^{\mathrm{A}} \mathcal{D}_{\delta \beta}^{\mathrm{A}}\right)\right]
\end{aligned}
$$

Поправка $\Delta \widetilde{\mathcal{D}}^{-+}$определяет неравновесную энергию решетки, связанную с избытком среднего числа фононов $n_{\mathrm{e}}=n\left(T_{\mathrm{e}}\right)$, разогретых электронами до температуры $T_{\mathrm{e}}$, над числом фононов $n$ с температурой среды $T$. Энергия, зависящая от $\Delta \overline{\mathcal{D}}^{-+}$, содержит поправку к энергии свободных фононов, оставшихся холодными после взаимодействия с электронами, а также, как будет видно из дальнейшего, поправку к энергии нулевых колебаний решетки.

Для того чтобы вычислить энергию $E$ с помошью формул (5)-(7) и (11)-(13), необходимо задать функции $|\psi(z)|^{2}$ и $\mathcal{D}_{\alpha \beta}^{\mathrm{R}, \mathrm{A}}$ в явном виде.

\section{3. ЗАПАЗДЫВАЮШИЕ И ОПЕРЕЖАЮШИЕ ФУНКЦИИ ГРИНА СВОБОДНЫХ АКУСТИЧЕСКИХ ФОНОНОВ В ПОЛУОГРАНИЧЕННОЙ СРЕДЕ}

Функции Грина, вычисленные в работе [13], являются решениями системы уравнений движения упругой среды

$$
\sum_{\gamma} \hat{L}_{\alpha \gamma}(X) \mathcal{D}_{\gamma \beta}\left(X, X_{1}\right)=\delta_{\alpha \beta} \delta\left(\mathbf{r}-\mathbf{r}_{1}\right) \delta\left(t-t_{1}\right) .
$$

Здесь лагранжиан $\hat{L}_{\alpha \gamma}(X)$ имеет вид

$$
\hat{L}_{\alpha \gamma}(X)=\delta_{\alpha \gamma} \frac{\partial^{2}}{\partial t^{2}}+\frac{1}{\rho} \sum_{\beta, \delta}\left(\frac{\partial}{\partial r_{\beta}} C_{\alpha \beta \gamma \delta}(z) \frac{\partial}{\partial r_{\delta}}+C_{\alpha \beta \gamma \delta}(z) \frac{\partial^{2}}{\partial r_{\beta} \partial r_{\delta}}\right),
$$


где $C_{\alpha \beta \gamma \delta}(z)$ - модули упругости. Одним граничным условием к уравнениям (14) в работе [13] является условие

$$
C_{\alpha \beta \gamma \delta}(z)=C_{\alpha \beta \gamma \delta} \Theta(z)
$$

с функцией включения $\Theta(z)$ равной 1 при $z \geqslant 0$ и 0 при $z<0$. Для кубических кристаллов модули упругости выражаются через $c_{\mathrm{t}}$ и $c_{1}-$ скорости t- и l-волн. Условие (16) означает, что вне образца со свободной поверхностью скорости звука пренебрежимо малы. Второе граничное условие к уравнениям (14) обусловлено в работе [13] затуханием их решений, запаздываюших во времени, при $z \rightarrow \infty$.

Решения уравнений (14) получены в работе [13] в $\omega, \mathbf{q}, z$-представлении и в системе координат, где ось $x$ совпадает с направлением $\mathbf{q}$. Эти функции связаны с функцией $\mathcal{D}_{\alpha \beta}\left(\omega, \mathbf{q}, z, z_{1}\right)$ формулой

$$
d_{\alpha \beta}\left(\omega, q, z, z_{1}\right)=\sum_{\gamma \delta} S_{\alpha \gamma}(\mathbf{q}) \mathcal{D}_{\gamma \delta}\left(\omega, \mathbf{q}, z, z_{1}\right) S_{\delta \beta}^{-1}(\mathbf{q}),
$$

где матрица перехода имеет вид

$$
S(\mathbf{q})=\frac{1}{q}\left(\begin{array}{ccc}
q_{x} & q_{y} & 0 \\
-q_{y} & q_{x} & 0 \\
0 & 0 & q
\end{array}\right) .
$$

Следствием преобразования (17) является независимость $d_{\alpha \beta}$ от направления вектора $\mathbf{q}$ и равенство $d_{\alpha \beta}=0 \mathrm{c} \alpha, \beta=y$ при $\alpha \neq \beta$. Отметим здесь, что функции $d_{\alpha \beta}\left(\omega, q, z, z_{1}\right)$ из работы [13] обладают следуюшим свойством по отношению к перестановкам $\alpha, \beta$ и $z, z_{1}$ :

$$
d_{\alpha \beta}\left(\omega, q, z, z_{1}\right)=(-1)^{\delta_{\alpha \beta}+1} d_{\beta \alpha}\left(\omega, q, z_{1}, z\right)
$$

Запаздьвание колебаний решетки во времени можно учесть непосредственно в решениях уравнения (14) в $\omega, \mathbf{q}, z$-представлении, заменив в них $\omega$ на $\omega+i \delta$, где $\delta \rightarrow 0$ [14]. Переход от функций $d_{\alpha \beta}^{\mathrm{A}}$ к функциям $d_{\alpha \beta}^{\mathrm{R}}$ в $\omega, \mathbf{q}, z$-представлении не сводится к простой замене $\omega+i \delta$ на $\omega-i \delta$, а затрагивает поляризационные индексы $\alpha, \beta$ и координаты $z, z_{1}$. По определению [12] имеем

$$
\mathcal{D}_{\alpha \beta}^{\mathrm{A}}\left(X, X_{1}\right)=\mathcal{D}_{\beta \alpha}^{\mathrm{R}}\left(X_{1}, X\right)
$$

Подвергнув (20) преобразованию Фурье и использовав (17), получаем, что

$$
d_{\alpha \beta}^{\mathrm{A}}\left(\omega, q, z, z_{1}\right)=d_{\beta \alpha}^{\mathrm{R} *}\left(\omega, q, z_{1}, z\right) .
$$

С учетом (19) это соотношение записывается в виде

$$
d_{\alpha \beta}^{\mathrm{A}}\left(\omega, q, z, z_{1}\right)=(-1)^{\delta_{\alpha \beta}+1} d_{\alpha \beta}^{\mathrm{R} *}\left(\omega, q, z, z_{1}\right) .
$$


В явном виде функции $d_{\alpha \beta}\left(\omega, q, z, z_{1}\right)$ весьма громоздки. Для дальнейшего использования они взяты из работы [13] при $z_{1}=z$ (см. приложение). Существенно, что функции $d_{\alpha \beta}\left(\omega, q, z, z_{1}\right)$ различны в трех областях $q$-пространства. Индексами $\mathrm{v}=1, \mathrm{t}, \mathrm{R}$ в (П.7)-(П.10) и далее обозначена принадлежность этих функций к одной из трех областей $q$. При $0 \leqslant q \leqslant \omega / c_{1}$ функция $d_{\alpha \beta}$ состоит из продольных и поперечных расходяшихся волн. В интервале $\omega / c_{1} \leqslant q \leqslant \omega / c_{\mathrm{t}}$ в $d_{\alpha \beta}$ остаются лишь t-волны, а l-волны затухают при $z \rightarrow \infty$. При $\omega / c_{\mathrm{t}} \leqslant q \leqslant \infty$ и $z \rightarrow \infty$ затухают обе объемные волны. Однако, как будет видно из дальнейшего, в области с такими $q$ образуются рэлеевские поверхностные волны [3], [4], [13].

\section{4. ЭНЕРГИЯ КОЛЕБАНИЙ ПОЛУОГРАНИЧЕННОЙ РЕШЕТКИ, ВЗАИМОДЕЙСТВУЮШИХ С ДВУМЕРНЫМ ГАЗОМ ПРИПОВЕРХНОСТНЫХ ГОРЯЧИХ ЭЛЕКТРОНОВ}

Функция распределения электронов в двумерном слое с толщиной $d \lesssim l$, лежашем вблизи поверхности на расстоянии $z_{0} \lesssim l$, может быть записана в виде

$$
|\psi(z)|^{2}=\delta(z) .
$$

После интегрирования выражений (12), (13) по $d z_{1}$ и $d z_{2}$ с функцией $\left|\psi\left(z_{1,2}\right)\right|^{2}$ из (23) величина $\Delta \mathcal{D}^{-+}$зависит от функций $\mathcal{D}_{\alpha \gamma}^{\mathrm{R}, \mathrm{A}}\left(\omega, \mathbf{q}, z, z_{1}\right)$ и $\mathcal{D}_{\delta \beta}^{\mathrm{R}, \mathrm{A}}\left(\omega, \mathbf{q}, z_{2}, z\right)$ при $z_{1}=$ $z_{2}=0$. Заметим теперь, что эти функции входят в $\Delta \mathcal{D}^{-+}$в виде характерных произведений

$$
\begin{aligned}
\sum_{\gamma, \delta} \hat{q}_{\gamma} \hat{q}_{\delta} \mathcal{D}_{\alpha \gamma}^{\mathrm{n}} \mathcal{D}_{\delta \beta}^{\mathrm{k}}= & \left(q d_{x x}^{\mathrm{n}}+i \frac{\partial d_{x z}^{\mathrm{n}}}{\partial z_{1}}\right)\left(q d_{x x}^{\mathrm{k}}+i \frac{\partial d_{x z}^{\mathrm{k}}}{\partial z_{2}}\right)- \\
& -\left(q d_{z x}^{\mathrm{n}}+i \frac{\partial d_{z z}^{\mathrm{n}}}{\partial z_{1}}\right)\left(q d_{z x}^{\mathrm{k}}+i \frac{\partial d_{z z}^{\mathrm{k}}}{\partial z_{2}}\right),
\end{aligned}
$$

где $d_{\alpha \beta}^{\mathrm{n}}=d_{\alpha \beta}^{\mathrm{n}}\left(\omega, q, z, z_{1}\right), d_{\alpha \beta}^{\mathrm{k}}=d_{\alpha \beta}^{\mathrm{k}}\left(\omega, q, z, z_{2}\right) \mathrm{c} \mathrm{n}, \mathrm{k}=\mathrm{R}, \mathrm{A}$. При выводе (24) использовалось преобразование, обратное (17), и свойство функций $d_{\alpha \beta}\left(\omega, q, z, z_{1}\right)$ относительно перестановок $\alpha, \beta$ и $z, z_{1}$ (19). Выражения (24) при $z_{1}=z_{2}=0$ можно значительно упростить, если воспользоваться следуюшими соотношениями [13]:

$$
\begin{aligned}
& q\left[1-2\left(\frac{c_{\mathrm{t}}}{c_{1}}\right)^{2}\right] d_{x x}(\omega, q, z, 0)+\left.i \frac{\partial}{\partial z_{1}} d_{x z}\left(\omega, q, z, z_{1}\right)\right|_{z_{1}=0}=0 \\
& q\left[1-2\left(\frac{c_{\mathrm{t}}}{c_{1}}\right)^{2}\right] d_{z x}(\omega, q, z, 0)+\left.i \frac{\partial}{\partial z_{1}} d_{z z}\left(\omega, q, z, z_{1}\right)\right|_{z_{1}=0}=0 .
\end{aligned}
$$

Отметим, что функции $d_{\alpha \beta}$ из работы [13], как и формулы (25), неприменимы при $\left(c_{\mathrm{t}} / c_{1}\right)^{2}=1 / 2$. Такое предельно большое отношение скоростей $c_{\mathrm{t}}$ и $c_{1}$ характеризует малореальные кристаллы с отсутствием поперечного сжатия при наличии продольного [3]. 
Теперь функция $\Delta \mathcal{D}^{-+}$, определяемая формулами (12), (13), с учетом связи функций $d_{\alpha \beta}^{\mathrm{A}}$ с $d_{\alpha \beta}^{\mathrm{R}}(22)$ и соотношений $(24),(25)$ записывается в более компактной форме,

$$
\begin{aligned}
& \Delta \widetilde{\mathcal{D}}_{\mathrm{v}}^{-+}(\omega, \mathbf{q}, z)=-\frac{2 i \lambda^{2} I_{2}}{(2 \pi)^{3} \rho}\left(n_{\mathrm{e}}-n\right) \Delta_{\mathrm{v} z}^{0}, \\
& \Delta \overline{\mathcal{D}}_{\mathrm{v}}^{-+}(\omega, \mathbf{q}, z)=-\frac{i \lambda^{2}}{(2 \pi)^{3} \rho} n\left(I_{2} \Delta_{\mathrm{v} z}^{+}+I_{1} \Delta_{\mathrm{v} z}^{-}\right),
\end{aligned}
$$

где $\Delta_{\mathrm{v} z}^{i}-$ безразмерные функции с $i=0,-,+$, равные при $\alpha=x, z$

$$
\begin{gathered}
\Delta_{\mathrm{v} z}^{0}=4 c_{\mathrm{t}}^{4} q^{2} \sum_{\alpha}\left|d_{\alpha x, \mathrm{v}}^{\mathrm{R}}\right|^{2} \\
\Delta_{\mathrm{v} z}^{+,-}=2 c_{\mathrm{t}}^{4} q^{2} \sum_{\alpha}(-1)^{\delta_{\alpha z}}\left[\left(d_{\alpha x, \mathrm{v}}^{\mathrm{R}}\right)^{2} \pm\left(d_{\alpha x, \mathrm{v}}^{\mathrm{R} *}\right)^{2}\right] .
\end{gathered}
$$

Энергия $\Delta E=\Delta \widetilde{E}+\Delta \bar{E}$, записанная с помошью формул (5), (6) и (26)-(29), принимает вид

$$
\begin{aligned}
& \Delta \widetilde{E}=\sum_{\mathrm{v}} \Delta \widetilde{E}^{\mathrm{v}}=\int_{-\infty}^{\infty} d \omega \omega^{3}\left[n_{\mathrm{e}}(\omega)-n(\omega)\right] \sum_{\mathrm{v}} A_{\mathrm{v}}^{+} \varphi_{\mathrm{v}}^{0}(\omega), \\
& \Delta \bar{E}=\sum_{\mathrm{v}} \Delta \bar{E}^{\mathrm{v}}=\int_{-\infty}^{\infty} d \omega \omega^{3} n(\omega) \sum_{\mathrm{v}} \sum_{j} A_{\mathrm{v}}^{j} \varphi_{\mathrm{v}}^{j}(\omega),
\end{aligned}
$$

где $j=+,-$, а

$$
A_{\mathrm{v}}^{+}=\frac{A_{\mathrm{v}}^{-}}{\sqrt{2}}\left(\frac{m c_{\mathrm{v}}^{2}}{\mu}\right)^{\frac{1}{2}}, \quad A_{\mathrm{v}}^{-}=\frac{L_{x} L_{y} \lambda^{2} m}{4 \pi^{3} \rho c_{1}^{4} c_{\mathrm{v}}} .
$$

Функции $\varphi_{\mathrm{v}}^{i}$ в (30), (31), определенные через переменные

$$
\zeta=\frac{q c_{\mathrm{v}}}{\omega}
$$

имеют вид

$$
\begin{aligned}
\varphi_{\mathrm{v}}^{0,+} & =\int_{a}^{b} d \zeta \Delta_{\mathrm{v}}^{0,+}(\zeta, \omega), \\
\varphi_{\mathrm{v}}^{-} & =-i \int_{a}^{b} d \zeta \zeta \Delta_{\mathrm{v}}^{-}(\zeta, \omega),
\end{aligned}
$$

где

$$
a=\left\{\begin{array}{ll}
0, & \mathrm{v}=1, \\
\frac{c_{\mathrm{t}}}{c_{\mathrm{l}}}, & \mathrm{v}=\mathrm{t}, \\
\frac{c_{\mathrm{R}}}{c_{\mathrm{l}}}, & \mathrm{v}=\mathrm{R},
\end{array} \quad b= \begin{cases}1, & \mathrm{v}=1, \mathrm{t}, \\
\infty, & \mathrm{v}=\mathrm{R} .\end{cases}\right.
$$

Функции $\Delta_{\mathrm{v}}^{i}$ в (34), (35) связаны с функциями (28) и (29) формулой

$$
\Delta_{\mathrm{v}}^{i}=\frac{\omega}{c_{\mathrm{v}}} \int_{0}^{L} d z \Delta_{\mathrm{v} z}^{i}(\omega, \zeta)
$$


В переменных $\zeta$ зависимость $\Delta_{\mathrm{v}}^{i}$ от $\omega$ определяется интегралом (см. (28), (29), (П.7)(П.11))

$$
\frac{\omega}{c_{\mathrm{v}}} \int_{0}^{L} d z \exp \left[\left( \pm a_{\mathrm{S}}-\beta_{\mathrm{s}}\right) \frac{\zeta z \omega}{c_{\mathrm{S}}}\right]= \begin{cases}\frac{c_{\mathrm{S}}\left(\beta_{\mathrm{S}} \mp i a_{\mathrm{S}}\right)}{c_{\mathrm{v}} \zeta\left(a_{\mathrm{S}}^{2}+\beta_{\mathrm{S}}^{2}\right)}, & L=L_{z}, \\ \frac{L_{z}^{0} \omega}{c_{\mathrm{v}}}, & L=L_{z}^{0},\end{cases}
$$

где $a_{\mathrm{s}}=\alpha_{\mathrm{t}} \pm \alpha_{1}, \alpha_{\mathrm{s}}$ при $\mathrm{s}=1, \mathrm{t}$. В (37) $L_{z} \gg c_{\mathrm{s}} / \omega, L_{z}^{0} \lesssim c_{\mathrm{s}} / \omega$. Имея это в виду, можно показать, что при $L=L_{z}$

$$
\varphi_{\mathrm{v}}^{0}=\varphi_{\mathrm{v}}+\frac{\bar{\varphi}_{\mathrm{s}} L_{z} \omega}{c_{\mathrm{S}}}, \quad \frac{d \varphi_{\mathrm{S}}^{j}}{d \omega}=0
$$

где $d \varphi_{\mathrm{s}} / d \omega=d \bar{\varphi}_{\mathrm{s}} / d \omega=0$. При $L=L_{z}^{0}$

$$
\varphi_{\mathrm{v}}^{i}=\frac{\bar{\varphi}_{\mathrm{v}}^{i} L_{z}^{0} \omega}{c_{\mathrm{v}}}
$$

где $d \bar{\varphi}_{\mathrm{s}}^{i} / d \omega=0$. Функции $\varphi_{\mathrm{R}}, \bar{\varphi}_{\mathrm{R}}, \varphi_{\mathrm{R}}^{i}$ содержат $\omega$ только в знаменателе, о чем будет сказано в дальнейшем.

\section{5. ЭНЕРГИЯ ОБЪЕМНЫХ КОЛЕБАНИЙ ПОЛУОГРАНИЧЕННОЙ РЕШЕТКИ С ГОРЯЧИМИ ЭЛЕКТРОНАМИ ВБЛИЗИ ЕЕ ПОВЕРХНОСТИ}

Выделим из выражений (30) и (31) суммарную энергию объемных t- и l-волн $\Delta E^{\mathrm{b}}$. Перейдем в выражении для $\Delta E^{\mathrm{b}}$ к интегрированию по положительным частотам, используя тож дество

$$
n(-\omega) \equiv-n(\omega)-1 .
$$

После простых преобразований с учетом зависимости $\varphi_{\mathrm{S}}^{j}$ от $\omega$ в виде (38) получаем, что $\Delta E^{\mathrm{b}}$ равна сумме энергий

$$
\begin{aligned}
& \Delta \widetilde{E}^{\mathrm{b}}=2 \int_{0}^{\infty} d \omega \omega^{3} n_{\mathrm{e}}(\omega) \sum_{\mathrm{s}} A_{\mathrm{s}}^{+} \varphi_{\mathrm{s}}, \\
& \Delta E_{\mathrm{T}}^{\mathrm{b}}=2 \int_{0}^{\infty} d \omega \omega^{3} n(\omega) \sum_{\mathrm{s}} A_{\mathrm{s}}^{-} \varphi_{\mathrm{s}}^{-}, \\
& \Delta E_{0}^{\mathrm{b}}=\int_{0}^{\Theta} d \omega \omega^{3} \sum_{\mathrm{s}} A_{\mathrm{s}}^{-} \varphi_{\mathrm{s}}^{-} .
\end{aligned}
$$

Переход к $\omega>0$ позволил выделить из $\Delta \bar{E}^{\mathrm{b}}$ энергию нулевых колебаний $\Delta E_{0}^{\mathrm{b}}$. В (41)-(43) учтено, что $c_{\mathrm{t}} \ll c_{\mathrm{l}}$. При этом условии все функции $\varphi_{\mathrm{s}}, \bar{\varphi}_{\mathrm{s}}, \varphi_{\mathrm{s}}^{j}$ и $\bar{\varphi}_{\mathrm{s}}^{i}$ являются фактически числами. Принимая во внимание, что $A_{\mathrm{s}}^{+} / A_{\mathrm{s}}^{-} \sim\left(m c_{\mathrm{s}}^{2} / \mu\right)^{1 / 2} \ll 1$, в $(42)$, (43) опущены члены, пропорциональные $A_{\mathrm{s}}^{+} \varphi_{\mathrm{s}}^{+}$. По этой же причине в $\Delta \widetilde{E}^{\mathrm{b}}$ не надо учитьвать слагаемые $n A_{\mathrm{s}}^{+} \varphi_{\mathrm{s}}$, поскольку они гораздо меньше слагаемых $n A_{\mathrm{s}}^{-} \varphi_{\mathrm{s}}^{-}$из $\Delta E_{\mathrm{T}}^{\mathrm{b}}$. 
Выражения для $\varphi_{\mathrm{s}}, \varphi_{\mathrm{s}}^{-}$довольно громоздки. Естественно, что $\varphi_{1}, \varphi_{1}^{-} \rightarrow 0$ при $c_{\mathrm{t}} / c_{1} \rightarrow 0$. Значения $\varphi_{\mathrm{t}} \simeq 0.8$ и $\varphi_{\mathrm{t}}^{-} \simeq 1$ полученыв результате численных расчетов. При этом величина $\varphi_{\mathrm{t}}$ определялась главными членами в $\Delta_{\mathrm{t}} \sim \sum_{\alpha}\left|a_{\alpha x, \mathrm{t}}^{1}\right|^{2}$. При вычислении $\varphi_{\mathrm{t}}^{-}$пришлось учитывать произведения коэффициентов $a_{\alpha x, \mathrm{~s}}^{\mathrm{s}}$ со всеми значениями $\mathrm{s}, \alpha$. Из сказанного следует, что неравновесная энергия объемных фононов $\Delta \widetilde{E}^{\mathrm{b}}$ зависит только от затухаюших продольных фононов с импульсами в интервале $\omega / c_{1} \leqslant q \leqslant \omega / c_{\mathrm{t}}$. $\mathrm{B}$ формировании энергий $\Delta E_{\mathrm{T}}^{\mathrm{b}}$ и $\Delta E_{0}^{\mathrm{b}}$ участвуют колебания обеих поляризаций с импульсами в том же интервале $q$. Подставив значения $\varphi_{\mathrm{t}}, \varphi_{\mathrm{t}}^{-}$в $(41)-(43)$, получаем, что

$$
\begin{aligned}
\Delta \widetilde{E}^{\mathrm{b}} & =\frac{2 \pi}{45} L_{x} L_{y} \frac{A}{p_{\mathrm{F}} c_{1}^{3}} T_{\mathrm{e}}^{4}, \\
\Delta E_{\mathrm{T}}^{\mathrm{b}} & =\frac{\pi}{30} L_{x} L_{y} \frac{A}{m c_{\mathrm{t}} c_{1}^{3}} T^{4}, \\
\Delta E_{0}^{\mathrm{b}} & =\frac{1}{16 \pi^{3}} L_{x} L_{y} \frac{A}{m c_{\mathrm{t}} c_{1}^{3}} \Theta^{4},
\end{aligned}
$$

где $p_{\text {Е }}=\sqrt{2 m \mu}$ и

$$
A=\frac{\lambda^{2} m^{2}}{\rho c_{1} \hbar}
$$

- безразмерньй параметр.

Формулы (44)-(46) справедливы при условии $L_{z} \gg c_{1} / \omega$ - наибольшем параметре длины в согласии с (37), где $\omega \sim T_{\mathrm{e}}, T$ и $\Theta$, поскольку интегралы (41)-(43) набираются соответственно при указанных значениях частот.

Рассмотрим пленки с толшинами $L_{z}^{0} \lesssim c_{\mathrm{s}} / \omega$ с одной свободной поверхностью и другой, вполне проницаемой для фононов. Как уже упоминалось (см. (39)), в этом случае $\varphi_{\mathrm{s}}^{i} \sim L_{z}^{0} \omega / c_{\mathrm{s}}$. В результате перехода к $\omega>0$ в формулах (30) и $(31)$ для $\Delta E^{\mathrm{b} 0}$ не равной нулю остается лишь энергия нулевых колебаний

$$
\Delta E_{0}^{\mathrm{b} 0}=\frac{-1}{2 \pi \cdot 10^{2}} L_{x} L_{y} L_{z}^{0} \frac{A}{m c_{\mathrm{t}}^{2} c_{1}^{3}} \Theta^{5} .
$$

Эта энергия сформирована волнами обеих поляризаций в том же интервале $q$, что и энергия больших образцов $\Delta E^{\mathrm{b}}$. Численные расчеты привели к значениям $\bar{\varphi}_{\mathrm{t}} \simeq-0.1$. Таким образом, термодинамическая энергия пленок с толшинами $L_{z}^{0} \lesssim c_{\mathrm{s}} / T$ может определяться или энергией рэлеевских волн, зависящей от температуры, или энергией возбуждения горячих электронов.

\section{6. НЕРАВНОВЕСНАЯ ЭНЕРГИЯ ПОВЕРХНОСТНЫХ ВОЛН}

Энергия $\Delta E^{\mathrm{R}}=\Delta \widetilde{E}^{\mathrm{R}}+\Delta \bar{E}^{\mathrm{R}}$ определяется формулами (30)-(36) $\mathrm{c} \mathrm{v}=\mathrm{R}$. Появление полюсов в функциях $d_{\alpha \beta, \mathrm{R}}^{\mathrm{R}}\left(\omega, q, z, z_{1}\right)$ свидетельствует о наличии поверхностных волн (R-фононов) с импульсами $q$ в интервале $\omega / c_{\mathrm{t}} \leqslant q \leqslant \infty$. Равенство нулю знаменателя этих функций (см. (П.9)-(П.11), [13]), а именно,

$$
\eta_{\mathrm{R}}=4 \beta_{\mathrm{t}} \beta_{1}-\gamma^{2}=0,
$$


совпадает с уравнением из работы [3], определяющим скорость рэлеевских волн $c_{\mathrm{R}}$ при $\omega=c_{\mathrm{R}} q$. Представим знаменатель функции $d_{\alpha \beta, \mathrm{R}}^{\mathrm{R}}$ в виде, аналогичном знаменателю запаздываюшей функции Грина объемных акустических фононов в неограниченном (двумерном) $q$-пространстве. Для этого разложим $\eta_{\mathrm{R}}$ в ряд по $\left[\left(c_{\mathrm{R}} q\right)^{2}-(\omega+i \delta)^{2}\right] /\left(c_{\mathrm{R}} q\right)^{2}$ (в работе [13] функция Грина $d_{\alpha \beta, \mathrm{R}}^{-1}$ уравнения (14) разложена в ряд по $\left.\left(c_{\mathrm{R}} q-\omega\right) / c_{\mathrm{R}} q\right)$. В переменных $\zeta=q c_{\mathrm{R}} / \omega$ функция $\eta_{\mathrm{R}}$ имеет вид

$$
\eta_{\mathrm{R}}=R\left[\zeta^{2}-\left(1+i \delta \omega^{-1}\right)^{2}\right] \zeta^{-2}=R \eta \zeta^{-2},
$$

где коэффициент

$$
R=2\left(\frac{c_{\mathrm{R}}}{c_{\mathrm{t}}}\right)^{2}\left\{\left[\left(\frac{c_{\mathrm{R}}}{c_{\mathrm{t}}}\right)^{2}-2\right]^{3}+4\left[\left(\frac{c_{\mathrm{t}}}{c_{\mathrm{l}}}\right)^{2}-2\left(\frac{c_{\mathrm{R}}}{c_{1}}\right)^{2}+1\right]\right\}\left[\left(\frac{c_{\mathrm{R}}}{c_{\mathrm{t}}}\right)^{2}-2\right]^{-2} .
$$

Перейдем в формулах (30), (31) для $\Delta E^{\mathrm{R}}$ к интегрированию по $\omega>0$. Для больших образцов с размерами $L_{z} \gg c_{\mathrm{R}} / \omega$ функции $\varphi_{\mathrm{R}}^{j}, \varphi_{\mathrm{R}}, \bar{\varphi}_{\mathrm{R}}$, согласно формулам (34), (35), (П.7)-(П.11), (28), (29), (38) и (50), содержат $\omega$ только в $\eta(\omega)$. Следовательно, $\varphi_{\mathrm{R}}$, $\bar{\varphi}_{\mathrm{R}}, \varphi_{\mathrm{R}}^{+}$- четные, а $\varphi_{\mathrm{R}}^{-}-$нечетная функции $\omega$. После соответствующих преобразований энергия рэлеевских волн становится равной сумме энергий

$$
\begin{aligned}
& \Delta \widetilde{E}^{\mathrm{R}}=2 A_{\mathrm{R}}^{+} \int_{0}^{\infty} d \omega \omega^{3}\left[n_{\mathrm{e}}(\omega)-n(\omega)\right] \varphi_{\mathrm{R}}(\omega), \\
& \Delta E_{\mathrm{T}}^{\mathrm{R}}=2 A_{\mathrm{R}}^{+} \int_{0}^{\infty} d \omega \omega^{3} n(\omega) \varphi_{\mathrm{R}}^{+}(\omega), \\
& \Delta E_{0}^{\mathrm{R}}=\int_{0}^{\Theta} d \omega \omega^{3}\left[A_{\mathrm{R}}^{+} \varphi_{\mathrm{R}}^{+}-A_{\mathrm{R}}^{-} \varphi_{\mathrm{R}}^{-}\right] .
\end{aligned}
$$

Интегралы $\varphi_{\mathrm{R}}, \varphi_{\mathrm{R}}^{j}$ могут быть приведены к виду

$$
\begin{aligned}
& \varphi_{\mathrm{R}}=\frac{1}{R^{2}} \int_{-\infty}^{\infty} d \zeta \frac{f(\zeta)}{|\eta(\zeta)|^{2}} \\
& \varphi_{\mathrm{R}}^{+}=\frac{1}{2 R^{2}} \int_{-\infty}^{\infty} d \zeta f(\zeta)\left[\frac{1}{\eta^{2}(\zeta)}+\frac{1}{\eta^{* 2}(\zeta)}\right] \\
& \varphi_{\mathrm{R}}^{-}=-\frac{i}{2 R^{2}} \int_{0}^{\infty} d \xi f(\xi)\left[\frac{1}{\eta^{2}(\xi)}-\frac{1}{\eta^{* 2}(\xi)}\right]
\end{aligned}
$$

где $\xi=\zeta^{2}$, а $\eta(\zeta), \eta(\xi)$ определяются формулой (50) при $\omega>0$. В функциях $f(\zeta), f(\xi)$ достаточно учесть главные члены, пропорциональные $\left|a_{z x, \mathrm{R}}^{\mathrm{t}}\right|^{2} \beta_{\mathrm{t}}^{-1},\left(a_{z x, \mathrm{R}}^{\mathrm{t}}\right)^{2} \beta_{\mathrm{t}}^{-1} \sim \nu^{-1}$, где

$$
\nu^{2}=1-\left(\frac{c_{\mathrm{R}}}{c_{\mathrm{t}}}\right)^{2} \ll 1
$$

В этом приближении

$$
f(\zeta)=\frac{\left(2 \zeta^{2}-1+\nu^{2}\right)^{2}}{\left(\zeta^{2}-1+\nu^{2}\right)^{\frac{1}{2}}}
$$


Дополнительная область интегрирования $-c_{\mathrm{R}} / c_{\mathrm{t}} \leqslant \zeta \leqslant c_{\mathrm{R}} / c_{\mathrm{t}}$, включенная в $\varphi_{\mathrm{R}}, \varphi_{\mathrm{R}}^{+}$, и область $0 \leqslant \xi \leqslant\left(c_{\mathrm{R}} / c_{\mathrm{t}}\right)^{2}$, добавленная в $\varphi_{\mathrm{R}}^{-}$(см. $\left.(34),(35)\right)$, не повлияют на величины $\varphi_{\mathrm{R}}, \varphi_{\mathrm{R}}^{j}$ с полюсами, лежащими вне этих областей. В результате интегралы (55) могут быть вычислены стандартным способом с помошью теоремы о вычетах. Они равны

$$
\varphi_{\mathrm{R}}=\frac{\pi \omega}{R^{2} \delta} f(1)=\frac{\pi \omega}{R^{2} \nu \delta}, \quad \varphi_{\mathrm{R}}^{+}=0, \quad \varphi_{\mathrm{R}}^{-}=\left.\frac{2 \pi}{R^{2}} \frac{\partial f(\xi)}{\partial \xi}\right|_{\xi=1}=\frac{\pi\left(8 \nu^{2}-1\right)}{\nu^{3} R^{2}}
$$

Как видно из (58), $\varphi_{\mathrm{R}} \rightarrow \infty$ при $\delta \rightarrow 0$. Эта расходимость возникла в $\varphi_{\mathrm{R}}$ от интегрирования членов, содержаших в знаменателе $(\zeta \mp 1 \mp i \delta / \omega)(\zeta \mp 1 \pm i \delta / \omega)$. Как и в работе [8], она может быть устранена заменой $\delta$ на конечную величину $1 / 2 \tau_{\mathrm{R}}$. Физический смысл $\tau_{\mathrm{R}}=\tau_{\mathrm{R}}(q)$ - время жизни рэлеевских фононов в состоянии с импульсом $\mathbf{q}$. Величина $1 / 2 \tau_{\mathrm{R}}$ характеризует ширину спектра $\mathrm{R}$-фононов. Степень зависимости $1 / \tau_{\mathrm{R}}$ от $q^{n}$, где $n$ - целое число, определяется механизмами рассеяния $\mathrm{R}$-фононов на каких-либо других частищах (например, примесях, шероховатостях поверхности, объемных фононах). Неравенство $\tau_{\mathrm{R}} \omega \gg 1$ является условием сушествования R-волны в виде стационарной частицы [14]. Его выполнение позволяет использовать в $\varphi_{\mathrm{R}}$ функции $\tau_{\mathrm{R}}\left(\zeta \omega / c_{\mathrm{R}}\right)$ при $|\zeta|=1$. В данной работе мы не будем конкретизировать вид $\tau_{\mathrm{R}}$. Выделим лишь из $\tau_{\mathrm{R}}$ частоту $\omega$ по формуле

$$
\tau_{\mathrm{R}}\left(\omega / c_{\mathrm{R}}\right)=\tau_{\mathrm{R}}(\Theta)(\Theta / \omega)^{n}
$$

Подставив $1 / 2 \tau_{\mathrm{R}}$ из $(59)$ вместо $\delta$ в $\varphi_{\mathrm{R}}$ и использовав $\varphi_{\mathrm{R}}, \varphi_{\mathrm{R}}^{j}$ из $(58)$ в формулах (52)-(54), получаем

$$
\begin{gathered}
\Delta \widetilde{E}^{\mathrm{R}}=\frac{\Gamma(5-n) \zeta(5-n) L_{x} L_{y} A}{\pi p_{\mathrm{F}} R^{2} c_{1}^{3} \nu} \tau(\Theta) \Theta^{n}\left(T_{\mathrm{e}}^{5-n}-T^{5-n}\right), \\
\Delta E_{\mathrm{T}}^{\mathrm{R}}=0, \\
\Delta E_{0}^{\mathrm{R}}=\frac{L_{x} L_{y} A(-8 \nu+1)}{16 \pi^{2} R^{2} \nu^{3} m c_{\mathrm{R}} c_{1}^{3}} \Theta^{4} .
\end{gathered}
$$

Здесь $A$ определяется формулой $(47), \Gamma(k), \zeta(k)$ - соответственно гамма- и дзета-функции. Степень $n$ в $\Delta \widetilde{E}^{\mathrm{R}}$ ограничена неравенством $n<4$. При $n \geqslant 4 \Delta \widetilde{E}^{\mathrm{R}} \rightarrow \infty$ и простая замена $\delta$ на $1 / 2 \tau_{\mathrm{R}}$ непригодна. Для обеспечения сходимости $\Delta \widetilde{E}^{\mathrm{R}}$ в этом случае требуется суммирование $1 / \tau_{\mathrm{R}}$ с шириной спектра, зависящей от $q$ в степени $n<4$, т.е. связанной с другим механизмом рассеяния R-волны [8].

В пленках с толщинами $L_{z} \lesssim c_{\mathrm{R}} / \omega$ функции $\varphi_{\mathrm{R}}^{i}$ выражаются формулой $(39)$, где $\bar{\varphi}_{\mathrm{R}}^{0}$, $\bar{\varphi}_{\mathrm{R}}^{+}-$четные, а $\bar{\varphi}_{\mathrm{R}}^{-}-$нечетная функции $\omega$. После перехода в формулах $(30),(31) \mathrm{c} v=\mathrm{R}$ к интегрированию по $\omega>0$ с учетом тождества (40) получаем, что

$$
\begin{aligned}
& \Delta \widetilde{E}^{\mathrm{R} 0}=0, \\
& \Delta E_{\mathrm{T}}^{\mathrm{R} 0}=\frac{2 L_{z}^{0} A_{\mathrm{R}}^{-}}{c_{\mathrm{R}}} \int_{0}^{\infty} d \omega \omega^{4} n(\omega) \bar{\varphi}_{\mathrm{R}}^{-}(\omega), \\
& \Delta E_{0}^{\mathrm{R} 0}=\frac{L_{z}^{0}}{c_{\mathrm{R}}} \int_{0}^{\Theta} d \omega \omega^{4}\left[A_{\mathrm{R}}^{-} \bar{\varphi}_{\mathrm{R}}^{-}-A_{\mathrm{R}}^{+} \bar{\varphi}_{\mathrm{R}}^{+}\right] .
\end{aligned}
$$


Функции $\bar{\varphi}_{\mathrm{R}}^{j}$, где $j=+,-$, могут быть записаны в виде $(55)$ с заменой $f(\zeta)$ на

$$
f_{0}=\left(2 \zeta^{2}-1+\nu^{2}\right)^{2} .
$$

Функции $f_{0}(\zeta)$ и $f_{0}\left(\xi=\zeta^{2}\right)$ при $c_{\mathrm{R}} \lesssim c_{\mathrm{t}} \ll c_{1}$ учитывают лишш главные члены в $\Delta_{\mathrm{R}}^{j} \sim$ $\left(a_{z x}\right)^{2} \beta_{\mathrm{t}}^{2} \sim 1$. Интегралы $\bar{\varphi}_{\mathrm{R}}^{j}$, вычисленные с помощью теоремы о вычетах, равны

$$
\bar{\varphi}_{\mathrm{R}}^{+}=0, \quad \bar{\varphi}_{\mathrm{R}}^{-}=\left.\frac{2 \pi}{R^{2}} \frac{\partial f_{0}(\xi)}{\partial \xi}\right|_{\xi=1}=\frac{4 \pi}{R^{2}} .
$$

Подставив (67) в (64), (65), получаем

$$
\begin{aligned}
\Delta E_{\mathrm{T}}^{\mathrm{R} 0} & =\frac{3 \cdot 2^{4} L_{x} L_{y} L_{z}^{0} A}{\pi^{2} R^{2} m c_{\mathrm{R}}^{2} c_{1}^{3}} T^{5}, \\
\Delta E_{0}^{\mathrm{R} 0} & =\frac{L_{x} L_{y} L_{z}^{0} A}{5 \pi^{2} R^{2} m c_{\mathrm{R}}^{2} c_{1}^{3}} \Theta^{5} .
\end{aligned}
$$

Энергию тонкой пленки $\Delta E_{\mathrm{T}}^{\mathrm{R} 0}$, представленную формулой (69), можно рассматривать как поправку к энергии свободных рэлеевских фононов в приповерхностном слое толщиной $z \lesssim c_{\mathrm{R}} / T$. Равенство $\Delta \widetilde{E}^{\mathrm{R} 0}=0$ при $z \lesssim c_{\mathrm{R}} / T_{\mathrm{e}}$ означает, что рэлеевские фононы в этой области не нагреваются до температуры $T_{\mathrm{e}}$. Поправки к энергии свободных нулевых рэлеевских колебаний существуют на любых расстояниях от поверхности. При этом $\Delta E_{0}^{\mathrm{R}} \sim \Delta E_{0}^{\mathrm{R} 0}$, если $z \sim c_{\mathrm{R}} / \nu \Theta$.

\section{7. ОБСУЖДЕНИЕ РЕЗУЛЬТАТОВ}

Полная средняя энергия полуограниченного кристалла с приповерхностным слоем горячих электронов представляет собой сумму колебательной энергии решетки (5) и энергии слоя горячих электронов

$$
E_{\mathrm{av}, \mathrm{e}}=E_{\mathrm{av}}+\Delta E+E^{\mathrm{e}}+\Delta E^{\mathrm{e}} .
$$

Здесь

$$
E_{\mathrm{av}}=\widetilde{E}^{\mathrm{b}}+\widetilde{E}^{\mathrm{R}}+E_{0}^{\mathrm{b}}+E_{0}^{\mathrm{R}}
$$

- энергия свободных колебаний полуограниченной решетки. Эта энергия вычислена в [7] для образцов различной толшины и приведена ниже в приложении (формулы (П.13)(П.16)). Энергия свободных электронов

$$
E^{\mathrm{e}}=\widetilde{E}^{\mathrm{e}}+E_{0}^{\mathrm{e}}
$$

где $\widetilde{E}^{\mathrm{e}}$ - энергия возбуждения, а $E_{0}^{\mathrm{e}}$ - энергия вырождения этих электронов в двумерном слое. Она вычислена в приложении ((П.17), (П.18)). В (70) можно пренебречь $\Delta E^{\mathrm{e}}$ - поправкой к $E^{\mathrm{e}}$, поскольку обе эти энергии зависят от $T_{\mathrm{e}}$. Полная средняя колебательная энергия полуограниченной решетки $\Delta E$ имеет вид

$$
\Delta E=\Delta \widetilde{E}^{\mathrm{b}}+\Delta \widetilde{E}^{\mathrm{R}}+\Delta E_{\mathrm{T}}^{\mathrm{b}}+\Delta E_{\mathrm{T}}^{\mathrm{R}}+\Delta E_{0}^{\mathrm{b}}+\Delta E_{0}^{\mathrm{R}} .
$$


Полученные для $\Delta E$ формулы верны при условии, что $\Delta E \ll E_{\mathrm{av}}$. При $T \ll T_{\mathrm{e}} \ll$ $\Theta$ в суммах (71) и (73) наибольшими являются энергии нулевых колебаний. Из формул (П.14), (П.16), (46), (48), (62) и (69) видно, что в толстых образцах $E_{0}^{\mathrm{b}} \gg E_{0}^{\mathrm{R}}$ и $\left|\Delta E_{0}^{\mathrm{b}}\right|>\Delta E_{0}^{\mathrm{R}}$, а в пленках $E_{0}^{\mathrm{R} 0} \gtrsim E_{0}^{\mathrm{b} 0}$ и $\left|\Delta E_{0}^{\mathrm{b} 0}\right| \gg \Delta E_{0}^{\mathrm{R} 0}$. Следовательно, условиями применимости формул $\Delta E$, полученных в данной работе, являются неравенства

$$
\frac{\left|\Delta E_{0}^{\mathrm{b}}\right|}{E_{0}^{\mathrm{b}}} \sim \frac{A}{m c_{1} L_{z}}\left(\frac{c_{\mathrm{t}}}{c_{1}}\right)^{2} \ll 1, \quad \frac{\left|\Delta E_{0}^{\mathrm{b} 0}\right|}{E_{0}^{\mathrm{R} 0}} \sim \frac{A R \nu \Theta}{m c_{\mathrm{t}}^{2}}\left(\frac{c_{\mathrm{R}}}{c_{\mathrm{l}}}\right)^{3} \ll 1 .
$$

Эти неравенства хорошо выполняются при обычных значениях параметров задачи: $\lambda \sim$ $3 \cdot 10^{15} \mathrm{c}^{-1}, \rho \sim 1 \mathrm{c} / \mathrm{cm}^{3}, T \sim m c_{1}^{2} \sim 10^{11} \mathrm{c}^{-1}, \Theta \sim \mu \sim 10^{13} \mathrm{c}^{-1}$ (с плотностью числа электронов $\rho_{\mathrm{e}} \sim 3 \cdot 10^{12} \mathrm{~cm}^{-2}$ и $\left.p_{\mathrm{F}} \sim 4 \cdot 10^{6} \mathrm{~cm}^{-1}\right), T_{\mathrm{e}} \sim 10^{12} \mathrm{c}^{-1}, \tau_{\mathrm{R}}\left(T_{\mathrm{e}}\right) T_{\mathrm{e}} \sim 10^{2}, c_{\mathrm{l}} \sim 3$. $10^{5} \mathrm{~cm} / \mathrm{c}, c_{\mathrm{t}} / c_{1} \sim 0.3, c_{\mathrm{R}} / c_{\mathrm{t}} \sim 0.9, \nu \sim 0.4, R \sim 3, A \sim 3 \cdot 10^{-2}$.

Выделим теперь из энергии $E_{\mathrm{av}, \mathrm{e}}$ наибольшую, зависящую от температуры. Для образцов толщиной $L_{z} \gg c_{1} / T$ и при принятых значениях параметров выполняются неравенства $\widetilde{E}^{\mathrm{t}} \gg \widetilde{E}^{\mathrm{e}} \gg \widetilde{E}^{\mathrm{R}}, \Delta \widetilde{E}^{\mathrm{R}} \gg \Delta \widetilde{E}^{\mathrm{b}} \gg \Delta E_{\mathrm{T}}^{\mathrm{b}}($ см. (П.13), (П.18), (44), (45), (60), $(61))$. Из этих соотношений следует, что наибольшими энергиями в суммах $(71)-(73)$ являются $\widetilde{E}^{\mathrm{t}}$ и $\Delta \widetilde{E}^{\mathrm{R}}$. Их отношение равно

$$
\frac{\Delta \widetilde{E}^{\mathrm{R}}}{\widetilde{E}^{\mathrm{t}}} \simeq \frac{L_{\mathrm{R}}}{L_{z}}
$$

где $L_{\mathrm{R}}=\bar{A} l_{\mathrm{R}} T_{\mathrm{e}} / T, \quad l_{\mathrm{R}}=\tau\left(T_{\mathrm{e}}\right) c_{\mathrm{R}}, \quad \bar{A}=\left(A / R^{2} \nu\right)\left(T_{\mathrm{e}} / p_{\mathrm{F}} c_{1}\right)\left(T_{\mathrm{e}} / T\right)^{3}\left(c_{\mathrm{t}} / c_{1}\right)^{2}$. При принятых значениях параметров $\bar{A} \sim 1, c_{\mathrm{l}} / T \sim 3 \cdot 10^{-6} \mathrm{~cm}, l_{\mathrm{R}} \sim 10^{-5} \mathrm{~cm}, L_{\mathrm{R}} \sim 10^{-4} \mathrm{~cm}$. Значения $L_{z} \sim L_{\mathrm{R}}$ являются пороговыми. В образцах толщиной $L_{z}<L_{\mathrm{R}}$ термодинамической энергией является неравновесная энергия рэлеевских волн $\Delta \widetilde{E}^{\mathrm{R}}(60)$. Если $L_{z}>L_{\mathrm{R}}$, то термодинамическая энергия описывается формулой (П.13) для энергии свободных поперечных фононов $\widetilde{E}^{\mathrm{t}}$.

Рассмотрим пленки толщиной $L_{z}^{0} \lesssim c_{\mathrm{t}} / T$. В них $\Delta \widetilde{E}^{\mathrm{b} 0}, \Delta E_{\mathrm{T}}^{\mathrm{b} 0}, \Delta \widetilde{E}^{\mathrm{R} 0}=0$ и, согласно (П.15), (П.18) и (69), $\widetilde{E}^{\mathrm{e}} \gg \widetilde{E}^{\mathrm{t} 0} \sim \widetilde{E}^{\mathrm{R} 0} \gg \Delta E_{\mathrm{T}}^{\mathrm{R} 0}$. Из этих неравенств следует, что термодинамической энергией пленок толшиной $L_{z}^{0} \lesssim c_{\mathrm{t}} / T \sim 10^{-6}$ см является $\widetilde{E}^{\mathrm{e}}$ - энергия возбуждения вырожденных электронов в двумерном газе (П.18).

\section{8. ЗАКЛЮЧЕНИЕ}

Итак, в работе получены условия, при которых термодинамическая энергия полуограниченных кристаллов с двумерным газом горячих электронов вблизи свободной поверхности определяется одним из трех видов энергии в зависимости от размера образца. Термодинамической энергией тонких пленок $\left(L_{z}^{0} \lesssim c_{\mathrm{t}} / T\right)$ является энергия возбуждения свободных электронов $\widetilde{E}^{\mathrm{e}} \sim T_{\mathrm{e}}^{2}$. В толстых пленках $\left(L_{\mathrm{R}} \gtrsim L_{z}>c_{\mathrm{t}} / T\right)$ термодинамической энергией становится неравновесная энергия рэлеевских волн $\Delta \widetilde{E}^{\mathrm{R}} \sim$ $T_{\mathrm{e}}^{5} \tau_{\mathrm{R}}\left(T_{\mathrm{e}}\right) \sim T_{\mathrm{e}}^{5-n}$. Эта энергия целиком обусловлена наличием свободной поверхности. Сушественно, что она зависит от тепловой длины пробега рэлеевских фононов, нагретых горячими электронами, $l_{\mathrm{R}}=\tau\left(T_{\mathrm{e}}\right) c_{\mathrm{R}}$. Термодинамическая энергия толстых образцов с тонким слоем горячих электронов определяется равновесной энергией свободных объемных фононов $\widetilde{E}^{\mathrm{b}} \sim T^{4}$. Отметим, что в случае равнораспределения горячих 
электронов по всему объему большого образца его термодинамическая энергия определяется неравновесной энергией объемных фононов $\sim T_{\mathrm{e}}^{5} \tau_{\mathrm{b}}\left(T_{\mathrm{e}}\right) \neq \Delta \widetilde{E}^{\mathrm{R}}\left(T_{\mathrm{e}}\right)$ [8]. Такое различие в зависимости термодинамической энергии кристаллов разного размера от температуры в значительной степени связана с характером распределения в них газа горячих электронов.

ПРИЛОЖЕНИЕ

А. Двумерный поляризационный оператор. Функции Келдыша свободных электронов удобно записать в виде [8]

$$
\begin{aligned}
& G^{--}(\varepsilon, \mathbf{p})=[1-n(\mathbf{p})] G^{\mathrm{R}}(\varepsilon, \mathbf{p})+n(\mathbf{p}) G^{\mathrm{A}}(\varepsilon, \mathbf{p})=-\left[G^{++}(\varepsilon, \mathbf{p})\right]^{*}, \\
& G^{+-}(\varepsilon, \mathbf{p})=2[1-n(\mathbf{p})] \operatorname{Im} G^{\mathrm{R}}(\varepsilon, \mathbf{p}), \\
& G^{-+}(\varepsilon, \mathbf{p})=-2 n(\mathbf{p}) \operatorname{Im} G^{\mathrm{R}}(\varepsilon, \mathbf{p}), \\
& G^{\mathrm{R}}(\varepsilon, \mathbf{p})=\left(\varepsilon-\varepsilon_{\mathbf{p}}+\mu+i \delta\right)^{-1}=\left[G^{\mathrm{A}}(\varepsilon, \mathbf{p})\right]^{*},
\end{aligned}
$$

где $\mathbf{p}=\left(p_{x}, p_{y}\right), \varepsilon_{\mathbf{p}}=\mathbf{p}^{2} / 2 m, m$ - масса электрона. В (П.1) распределение Ферми

$$
n(\mathbf{p})=\left[\exp \left(\frac{\varepsilon_{\mathbf{p}}-\mu}{T_{\mathrm{e}}}\right)+1\right]^{-1}
$$

умножается на $\operatorname{Im} G^{\mathrm{R}, \mathrm{A}}=\mp \pi \delta\left(\varepsilon-\varepsilon_{\mathbf{p}}+\mu\right)$. Поэтому, в отличие от распределения Планка $n(\omega)$ (см. (4)), в (П.1) $n(\varepsilon)=n(\mathbf{p})$.

Вычислим поляризационные части $\Pi^{i j}(\omega, \mathbf{q})$, проинтегрировав (10) вначале по $d \varepsilon \mathrm{c}$ помошью теоремы о вычетах [14]. Перед интегрированием по $d \mathbf{p}$ воспользуемся тождеством, справедливым при $\varepsilon_{\mathbf{p}}=\omega+\varepsilon_{\mathbf{p}-\mathbf{q}}$,

$$
n_{\mathrm{e}}(\omega) \equiv \frac{n(\mathbf{p})[1-n(\mathbf{p})]}{n(\mathbf{p}-\mathbf{q})-n(\mathbf{p})} .
$$

В результате получаем, что двумерные поляризационные части $\Pi^{i j}(\omega, \mathbf{q})$ имеют формально одинаковый вид с трехмерными [8], а именно,

$$
\begin{aligned}
& \Pi^{--}(\omega, \mathbf{q})=i I_{1}+\left(2 n_{\mathrm{e}}+1\right) I_{2}=-\left[\Pi^{++}(\omega, \mathbf{q})\right]^{*} \\
& \Pi^{+-}(\omega, \mathbf{q})=2\left(n_{\mathrm{e}}+1\right) I_{2} \\
& \Pi^{-+}(\omega, \mathbf{q})=2 n_{\mathrm{e}} I_{2}
\end{aligned}
$$

Двумерные интегралы $I_{1}$ и $I_{2}$ в $2 p_{\text {F }}$ раз меньше соответствуюших трехмерных и даются выражениями

$$
\begin{aligned}
& I_{1}=2 \pi f d \mathbf{p} \frac{n(\mathbf{p}-\mathbf{q})-n(\mathbf{p})}{\omega+\varepsilon_{\mathbf{p}-\mathbf{q}}-\varepsilon_{\mathbf{p}}}=-4 \pi^{2} m, \\
& I_{2}=2 \pi^{2} \int d \mathbf{p}[n(\mathbf{p}-\mathbf{q})-n(\mathbf{p})] \delta\left(\omega+\varepsilon_{\mathbf{p}-\mathbf{q}}-\varepsilon_{\mathbf{p}}\right)=\frac{4 \pi^{2} m^{2} \omega}{p_{\mathrm{F}} q}
\end{aligned}
$$

при $\mu>T_{\mathrm{e}}$, когда электроны вырожденны. 
Б. Запаздывающие функции Грина уравнений движения упругих колебаний полуограниченной решетки. Решениями уравнений движения (14) с учетом запаздывания во времени в $\omega, \mathbf{q}, z$-представлении являются функции $Г$ рина $d_{\alpha \beta}(\omega, q$, $\left.z, z_{1}\right)$, вычисленные в работе [13], при замене в них $\omega$ на $\omega+i \delta$. Запаздываюшие функции Грина $d_{\alpha \beta}^{\mathrm{R}}\left(\omega, q, z, z_{1}\right)$ с $\beta=x$ и $z_{1}=0$, используемые в этой работе, имеют вид

$$
d_{\alpha x, \mathrm{v}}^{\mathrm{R}}(\omega, q, z, 0)=-\frac{2 \alpha_{\mathrm{v}}^{\mathrm{t}} \gamma}{c_{\mathrm{t}}^{2} q \eta_{\mathrm{v}}} \sum_{\mathrm{s}=\mathrm{t}, 1} \exp \left(-\alpha_{\mathrm{v}}^{\mathrm{s}} z q\right) a_{\alpha x, \mathrm{v}}^{\mathrm{s}}
$$

где

$$
\begin{aligned}
& a_{x x, \mathrm{v}}^{\mathrm{t}}=\frac{1}{2}, \quad a_{x x, \mathrm{v}}^{1}=\frac{1}{\gamma}, \quad a_{z x, \mathrm{v}}^{\mathrm{t}}=\frac{i}{2 \alpha_{\mathrm{v}}^{\mathrm{t}}}, \quad a_{z x, \mathrm{v}}^{1}=\frac{i \alpha_{\mathrm{v}}^{1}}{\gamma}, \\
& \eta_{\mathrm{v}}=4 \varepsilon_{\mathrm{v}}-\gamma^{2}, \quad \varepsilon_{\mathrm{v}}=\alpha_{\mathrm{v}}^{1} \alpha_{\mathrm{v}}^{\mathrm{t}}, \quad \gamma=\alpha_{\mathrm{t}}^{2}-1, \\
& \alpha_{\mathrm{v}}^{\mathrm{t}}=\left\{\begin{array}{ll}
-i \alpha_{\mathrm{t}}, & \mathrm{v}=1, \mathrm{t}, \\
\beta_{\mathrm{t}}, & \mathrm{v}=\mathrm{R},
\end{array} \quad \alpha_{\mathrm{v}}^{1}= \begin{cases}-i \alpha_{\mathrm{l}}, & \mathrm{v}=1, \\
\beta_{1}, & \mathrm{v}=\mathrm{t}, \mathrm{R},\end{cases} \right. \\
& \alpha_{\mathrm{S}}=\left[\left(\frac{\omega+i \delta}{c_{\mathrm{s}} q}\right)^{2}-1\right]^{\frac{1}{2}}, \quad \beta_{\mathrm{S}}=\left(1-\frac{\omega}{c_{\mathrm{s}} q}\right)^{\frac{1}{2}} \text {. }
\end{aligned}
$$

Условия затухания $d_{\alpha x, \mathrm{v}}^{\mathrm{R}}$ при $z \rightarrow \infty$ (затем $\delta \rightarrow 0$ ) определяются неравенствами

$$
\left|\operatorname{Re} i \alpha_{\mathrm{s}} q z\right| \gg 1, \quad \beta_{\mathrm{s}} q z \gg 1 .
$$

В. Полная средняя энергия колебаний полуограниченной решетки. Средняя энергия полуограниченной решетки $E_{\mathrm{av}}=E^{\mathrm{b}}+E^{\mathrm{R}}$, вычисленная в работе [7], при $L_{z} \gg c_{1} / T$ определяется формулами

$$
\begin{aligned}
\widetilde{E}^{\mathrm{b}}=\frac{\pi^{2}}{30} L_{x} L_{y} L_{z}\left(\frac{2}{c_{\mathrm{t}}^{3}}+\frac{1}{c_{1}^{3}}\right) T^{4}, & \widetilde{E}^{\mathrm{R}} \simeq \frac{\pi}{10 R \nu^{2} c_{\mathrm{R}}^{2}} L_{x} L_{y} T^{3}, \\
E_{0}^{\mathrm{b}}=\frac{1}{16 \pi^{2}} L_{x} L_{y} L_{z}\left(\frac{2}{c_{\mathrm{t}}^{3}}+\frac{1}{c_{1}^{3}}\right) \Theta^{4}, & E_{0}^{\mathrm{R}} \simeq \frac{1}{12 \pi R \nu^{2} c_{\mathrm{R}}^{2}} L_{x} L_{y} \Theta^{3} .
\end{aligned}
$$

Выражения для $\widetilde{E}^{\mathrm{b}}, E_{0}^{\mathrm{b}}$ совпадают с классическими из книги [1].

Энергия пленок толщиной $L_{z}^{0} \lesssim c_{\mathrm{t}} / T$ имеет вид

$$
\begin{aligned}
\widetilde{E}^{\mathrm{b} 0}=\frac{\pi^{2}}{10 c_{\mathrm{t}}^{3}} L_{x} L_{y} L_{z}^{0} T^{4}, & \widetilde{E}^{\mathrm{R} 0} \simeq \frac{\pi^{3}}{30 R \nu c_{\mathrm{R}}^{3}} L_{x} L_{y} L_{z}^{0} T^{4}, \\
E_{0}^{\mathrm{b} 0}=\frac{3}{16 \pi^{2} c_{\mathrm{t}}^{3}} L_{x} L_{y} L_{z}^{0} \Theta^{4}, & E_{0}^{R 0} \simeq \frac{1}{16 \pi R \nu c_{\mathrm{R}}^{3}} L_{x} L_{y} L_{z}^{0} \Theta^{4} .
\end{aligned}
$$

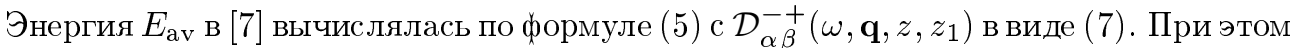
использовалось преобразование (17) и функции $d_{\alpha \beta}\left(\omega, q, z, z_{1}\right)$ из работы [13] при $z_{1}=$ $z$. Энергии $E^{\mathrm{R}}$ и $E^{\mathrm{b} 0}$ волн здесь приведены при $c_{\mathrm{t}} / c_{1} \ll 1$. 
Г. Полная средняя энергия двумерного слоя электронного газа. Средняя энергия свободных электронов, распределенных в слое, параллельном поверхности, согласно определениям из работ [11], [14], [7], [8] задается соотношением

$$
E^{\mathrm{e}}=-\frac{2 i L_{x} L_{y}}{(2 \pi)^{3}} \int d \mathbf{p} \frac{p^{2}}{2 m} \int_{-\infty}^{\infty} d \varepsilon \int_{0}^{L} d z|\psi(z)|^{2} G^{-+}(\varepsilon, \mathbf{p})
$$

Здесь функция $G^{-+}(\varepsilon, \mathbf{p})$ определяется формулами (П.1), (П.2), а распределение электронов $|\psi(z)|^{2}$ в двумерном слоевблизи поверхности - формулой (23). Энергия $E^{\mathrm{e}}$ в (П.17) распадается на энергию возбуждения $\widetilde{E}^{\mathrm{e}}$ и энергию вырождения $E_{0}^{\mathrm{e}}$,

$$
\widetilde{E}^{\mathrm{e}}=\frac{\pi}{6} L_{x} L_{y} m T_{\mathrm{e}}^{2}, \quad E_{0}^{\mathrm{e}}=\frac{2}{\pi} L_{x} L_{y} m \mu^{2}
$$

при $\mu>T_{\mathrm{e}}$, когда электроны вырожденны.

Благодарности. В заключение выражаю свою признательность И.Б. Левинсону за предложенную тему исследования и полезные советы. Я также благодарна А. С. Простневу за внимание и помощь в работе. Эта работа поддерживалась Российским фондом фундаментальных исследований (проект № 99-03-32101).

\section{Список литературы}

[1] Л. Д. Ландау, Е. М. Лифииц. Статистическая физика. Ч. 1. М.: Наука, 1976.

[2] В. Л. Бонч-Бруевич, С. Г. Калашников. Физика полупроводников. М.: Наука, 1977.

[3] Л. Д. Ландау, Е. М. Лифииц. Теория упругости. М.: Наука, 1987.

[4] W. Rayleigh. London. Math. Soc. Proc. 1887. V. 17. P. 4.

[5] V. Dupuis, R. Maro, L. Onsager. Journ. Chem. Phys. 1960. V. 33. P. 1452.

[6] A. Марадудин. Дефекты и колебательный спектр в кристаллах. М.: Мир, 1968.

[7] Л. А. Зайцева. Хим. физика. 2000. Т. 19. С. 3.

[8] Л. А. Зайцева. ТМФ. 1998. Т. 115. С. 289.

[9] М. И. Каганов, И. М. Лифииц, Л. В. Танатаров. ЖЭТФ. 1956. Т. 31. С. 232.

[10] С. М. Бадалян, И. Б. Левинсон. ФТТ. 1988. Т. 30. С. 2764.

[11] Л. В. Келдьи. ЖЭТФ. 1964. Т. 47. С. 1515.

[12] Е. М. Лифиич, Л. П. Питаевский. Физическая кинетика. М.: Наука, 1979.

[13] A. A. Maradudin, D. L. Mills. Annals of Physics. 1976. V. 100. P. 262.

[14] А.А. Абрикосов, Л. П. Горьков, И.Е. Дзялошинский. Методы квантовой теории поля в статистической физике. М.: Наука, 1962. 